\title{
NUMERICAL SIMULATION OF FLOW FAILURE OF GEOMATERIALS BASED ON FLUID DYNAMICS
}

\author{
Shuji Moriguchi ${ }^{\mathrm{i})}$, Atsushi Yashima ${ }^{\mathrm{i})}$, Kazuhide Sawada ${ }^{\mathrm{i})}$, Ryosuke Uzuoka ${ }^{\mathrm{ii})}$ and Masatoshi Ito ${ }^{\mathrm{i})}$
}

\begin{abstract}
A numerical method is developed for the prediction of large deformations associated with a geomaterial flow during the occurrences of such geo-disasters as landslides and slope failures. The geomaterial is modeled as a viscous fluid, where a Bingham type constitutive model is proposed based on Coulomb's failure criterion and the viscosity is derived from the cohesion and friction angle. Numerical experiments on a 2D gravitational flow of a geomaterial column show that the constitutive model performs well. For solving Navier-Stokes's equations, Constrained Interpolated Profile (CIP) scheme is utilized, which is able to efficiently treat solid, liquid and gas together and has been successfully applied to problems in fluid dynamics. To prevent numerical instabilities due to a large Bingham viscosity, an implicit calculation procedure is proposed to calculate the viscous forces in the CIP. The method is finally used to simulate an earthquake-induced landslide that took place on May 26th, 2003 in the Northern part of Miyagi Prefecture in Japan. Results from soil-slump tests on the samples from the landslide site are used for determining the parameters in the simulation. Good agreements between the calculated and the observed results are obtained.
\end{abstract}

Key words: Bingham model, CIP, fluid dynamics, geo-disaster, landslide, large deformation, shear strength (IGC: E0)

\section{INTRODUCTION}

Geo-disasters accompanied by large deformations such as landslides and slope failures have worldwide been implicated with loss of life, damage to natural resources and damage to important infrastructures such as roads and bridges. Based on data of topography, geology, hydrology, seismology and records of past occurrences for a given slope, several attempts have been made to determine the probability occurrence of such incidents and to devise hazard maps. These attempts have primarily aimed at developing a means of predicting where and when a geo-disaster would take place. On the other hand, in order to establish efficient hazard maps, it is equally important to predict the extent of ground displacement, velocity and elapsed time once such incidents occur. The prime objective of this paper is to devise a numerical method for the prediction of large deformation during the occurrence of a landslide, which will be very useful in the preparation of a hazard map.

There are some traditional numerical methods for deformation and failure of geomaterials in the framework of continuum mechanics, such as Finite Element Method (FEM), Finite Difference Method (FDM) and Boundary Element Method (BEM). These methods have been successfully implemented and many interesting results have been reported. Large deformation problems, however, produce numerical instabilities due to excessive distortion of a mesh. To overcome this problem, a lot of methods have been proposed for large deformation problems up to now. Good examples are Up-dated Lagrangian methods and Arbitrary Lagrangian-Eulerian method (ALE) (Huerta and Liu, 1988), which are similar to the traditional FEM methods at the point of using finite element mesh. In these methods, the mesh is updated step by step. As opposed to these methods, Element Free Galerkin Method (EFGM), proposed by Belytschko and $\mathrm{Lu}$ (1994), does not use finite element mesh. It is possible to apply these methods to large deformation problems. It is, however, difficult to solve problems with extremely large deformation like soil avalanche and slope failure flow by these methods.

Different numerical methods have been proposed to predict the behavior of geomaterials subjected to flow. For example, Uzuoka et al. $(1998,2000)$ and Hadush et al. (2000) treated liquefied soil undergoing ground flow as a Bingham fluid and developed fluid dynamics based numerical methods. Using results from shaking table tests (Hamada and Wakamatsu, 1998; Sakaguchi and Yoshida, 1998), they showed that the methods were capable of reproducing the time histories of displacement and velocity of the liquefaction induced ground flow. The limitation of the method based on fluid dynamics is that the history characteristic of geomaterials cannot be taken into consideration because the numerical scheme is based on Eulerian scheme. For this reason, the influence of

i) Gifu University, Japan (sawada@cc.gifu-u.ac.jp).

ii) Tohoku University, Japan.

The manuscript for this paper was received for review on March 1, 2004; approved on March 11, 2005.

Written discussions on this paper should be submitted before November 1, 2005 to the Japanese Geotechnical Society, 4-38-2, Sengoku, Bunkyo-ku, Tokyo 112-0011, Japan. Upon request the closing date may be extended one month. 
strain softening, hardening and dilatancy cannot be taken into consideration.

In this work, a fluid dynamics based numerical method is developed to predict landslide movement, in which the ground is treated as a viscous fluid. Special attentions are given to representing the viscous behavior of the geomaterial with universally accepted shear strength properties and to solving the Navier-Stoke's equations with a scheme that treats multi-phases (solid, liquid and gas) together. A Bingham type constitutive model is formulated based on Coulomb's failure criterion, in which the cohesion and friction angle of the soil are the only parameters required to estimate the viscosity of the geomaterial. Since both cohesion and friction angle are easily obtainable parameters, this model is particularly useful for practical engineers. CIP (Constrained Interpolated Profile) scheme (Yabe and Aoki, 1991) is employed for solving the Navier-Stokes equations. To avoid numerical instabilities due to a large gradient of viscosity, an implicit calculation procedure is recommended to calculate the viscous forces in CIP.

The numerical method developed in this paper is first used to simulate pressure distribution in a very simple analytical condition. Using numerical results of this simulation, the effectiveness of the implicit scheme proposed in this paper is discussed. The method is then applied to simulate a 2-dimensional gravitational flow of different materials in order to investigate the deformation behavior and performance of the constitutive model. Moreover, in order to obtain numerical parameters for simulations of a real landslide, the method is applied to simulate a soil-slump laboratory test. Based on the parameters obtained in the soil-slump simulation, the numerical method is finally used to simulate an earthquake-induced landslide that took place on May 26th, 2003 in the Northern part of Miyagi Prefecture, Japan. Simulated results of ground flow velocity, displacement and elapsed time are compared with observed results.

\section{CONSTITUTIVE MODEL}

In this paper, the geomaterial subjected to flow is modeled as a non-Newtonian fluid, which is considered to have an internal structure that collapses above a certain yield stress. For a non-Newtonian fluid with a yield strength, the shear stress $\tau$ to shear strain rate $\dot{\gamma}$ relation is given as

$$
\tau=\eta \dot{\gamma}+\tau_{\min }
$$

where $\eta$ is the viscosity and $\tau_{\min }$ the yield strength. The above equation is generally termed as a Bingham model. For shear stresses below the yield stress, a Bingham fluid behaves as a rigid body and does not deform but when the shear stress surpasses the yield stress, flow failure occurs resulting in very large deformations. The following Coulomb equation is widely accepted for expressing the shear strength of a given soil.

$$
s=c+\sigma \tan \phi
$$

where $s$ is the shear strength, $c$ the cohesion, $\sigma$ the normal stress and $\phi$ the friction angle. In this study, we use this basic failure criterion to formulate a constitutive model for geomaterials subjected to flow. Substituting the shear strength $s$ in Eq. (2) by the yield strength $\tau_{\min }$ in Eq. (1) and representing the normal stress $\sigma$ by the hydraulic pressure $p$, we propose the following Bingham model:

$$
\tau=\eta \dot{\gamma}+c+p \tan \phi
$$

This model is simple to understand and requires only two easily obtainable parameters $c$ and $\phi$, hence would be convenient for engineering practice. Since the NavierStoke's equations, which are the governing equations of fluid motion, are developed based on a Newtonian fluid, an equivalent Newtonian viscosity is used to express the Bingham viscosity. Dividing Eq. (3) by the shear strain rate $\dot{\gamma}$ and denoting the equivalent Newtonian viscosity as $\eta^{\prime}$, we have

$$
\eta^{\prime}=\eta+\frac{c+p \tan \phi}{\dot{\gamma}}
$$

The shear strain rate is calculated using the following equation,

$$
\dot{\gamma}=\sqrt{\frac{1}{2} \dot{e}_{\mathrm{ij}} \dot{e}_{\mathrm{ij}}}
$$

where $\dot{e}_{\mathrm{ij}}$ is the second invariant of the deviatoric strain rate tensor. By using Eq. (4), an infinite equivalent viscosity coefficient arises when shear strain rate equals to zero. In order to avoid such infinite value that cannot be treated in numerical simulations, a maximum value for the equivalent viscosity coefficient is determined as below,

$$
\begin{array}{lll}
\eta^{\prime}=\eta_{0} \dot{\gamma}+\frac{\tau_{y}}{\dot{\gamma}} & \text { if } & \eta^{\prime}<\eta_{\max } \\
\eta^{\prime}=\eta_{\max } & \text { if } & \eta^{\prime}<\eta_{\max }
\end{array}
$$

where $\eta_{\max }$ is maximum equivalent viscosity coefficient. At the same time, minimum value of equivalent viscosity coefficient is determined by $\eta$. Uzuoka (2000) used a small value $(0.1$ or $1.0 \mathrm{~Pa} \cdot \mathrm{s})$, and it was found that numerical results do not depend on the value of $\eta$. Therefore, $1.0 \mathrm{~Pa} \cdot \mathrm{s}$ is used for the value of $\eta$ in this study.

\section{NUMERICAL METHOD}

\section{Governing Equation}

In this study, the following equations are used as governing equations.

$$
\begin{aligned}
& \frac{\partial u_{\mathrm{i}}}{\partial t}+u_{\mathrm{j}} \frac{\partial u_{\mathrm{i}}}{\partial x_{\mathrm{j}}}=-\frac{1}{\rho} \frac{\partial p}{\partial x_{\mathrm{i}}}-\frac{2}{3} \frac{1}{\rho} \frac{\partial}{\partial x_{\mathrm{i}}}\left(\eta^{\prime} \frac{\partial u_{\mathrm{k}}}{\partial x_{\mathrm{k}}}\right) \\
&+\frac{1}{\rho} \frac{\partial}{\partial x_{\mathrm{j}}}\left[\eta^{\prime}\left(\frac{\partial u_{\mathrm{i}}}{\partial x_{\mathrm{j}}}+\frac{\partial u_{\mathrm{j}}}{\partial x_{\mathrm{i}}}\right)\right]+g_{\mathrm{i}} \\
& \frac{\partial \rho}{\partial t}+u_{\mathrm{j}} \frac{\partial \rho}{\partial x_{\mathrm{j}}}=-\rho\left(\frac{\partial u_{\mathrm{k}}}{\partial x_{\mathrm{k}}}\right) \\
& \frac{\partial p}{\partial t}+u_{\mathrm{j}} \frac{\partial p}{\partial x_{\mathrm{j}}}=-\rho C_{\mathrm{s}}^{2}\left(\frac{\partial u_{\mathrm{k}}}{\partial x_{\mathrm{k}}}\right)
\end{aligned}
$$


where $\rho$ is the density, $u$ is the velocity, $p$ is the pressure, $C_{\mathrm{s}}$ is the speed of sound and $g$ stands for gravity. Equation (7) describes the Navier-Stokes equation. Spatial gradient of viscosity is considered in Eq. (7), because equivalent viscosity $\eta^{\prime}$ changes with space. Equations (8) and (9) show the governing equations of density and pressure, respectively.

In this study, the CIP scheme is used to solve the governing equations because the method can correctly describe flow behaviors at interfaces of multi-fluids. In CIP, density functions (color functions) $\phi_{\mathrm{i}}$ are used to distinguish different kinds of materials (Xiao, 1999). Density functions are defined in each mesh. If $\phi_{\mathrm{i}}=1$, the mesh is filled up with the material $i$, and if $\phi_{\mathrm{i}}=0$, there is no material $i$ in the mesh. Using the density functions, the viscosity coefficient and the sound speed are calculated as follows,

$$
\begin{array}{ll}
\eta=\Sigma \phi_{\mathrm{i}} \eta_{\mathrm{i}} & \left(0 \leq \phi_{\mathrm{i}} \leq 1\right) \\
C_{\mathrm{s}}=\Sigma \phi_{\mathrm{i}} C_{\mathrm{si}} & \left(0 \leq \phi_{\mathrm{i}} \leq 1\right)
\end{array}
$$

The color functions are then predicted according to following equation,

$$
\frac{\partial \phi}{\partial t}+u_{\mathrm{j}} \frac{\partial \phi}{\partial x_{\mathrm{j}}}=0
$$

The key issues in CIP are in the representation of advection terms and split treatment of other terms. In this method, by using the time splitting technique, the governing equations are deduced into two phases, namely non-advection phase and advection phase.

\section{Non-advection Phase}

$$
\begin{aligned}
& \frac{\partial u_{\mathrm{i}}}{\partial t}=-\frac{1}{\rho} \frac{\partial p}{\partial x_{\mathrm{i}}}-\frac{2}{3} \frac{1}{\rho} \frac{\partial}{\partial x_{\mathrm{i}}}\left(\eta^{\prime} \frac{\partial u_{\mathrm{k}}}{\partial x_{\mathrm{k}}}\right) \\
&+\frac{1}{\rho} \frac{\partial}{\partial x_{\mathrm{j}}}\left[\eta^{\prime}\left(\frac{\partial u_{\mathrm{i}}}{\partial x_{\mathrm{j}}}+\frac{\partial u_{\mathrm{j}}}{\partial x_{\mathrm{i}}}\right)\right]+g_{\mathrm{i}} \\
& \frac{\partial \rho}{\partial t}=-\rho\left(\frac{\partial u_{\mathrm{k}}}{\partial x_{\mathrm{k}}}\right) \\
& \frac{\partial p}{\partial t}=-\rho C_{\mathrm{s}}^{2}\left(\frac{\partial u_{\mathrm{k}}}{\partial x_{\mathrm{k}}}\right)
\end{aligned}
$$

Equations (13), (14) and (15) show non-advection phases of the governing equations. It is widely accepted that the governing equation in this phase can be solved by common finite difference schemes. The Eulerian type finite difference scheme is used in this research and the approximations of Eqs. (13), (14) and (15) are given as:

$$
\begin{gathered}
\frac{u_{\mathrm{i}}^{*}-u_{\mathrm{i}}^{\mathrm{n}}}{\Delta t}=-\frac{1}{\rho} \frac{\partial p^{\mathrm{n}}}{\partial x_{\mathrm{i}}}-\frac{2}{3} \frac{1}{\rho^{\mathrm{n}}} \frac{\partial}{\partial x_{\mathrm{i}}}\left(\eta^{\prime} \frac{\partial u_{\mathrm{k}}^{*}}{\partial x_{\mathrm{k}}}\right) \\
+\frac{1}{\rho^{\mathrm{n}}} \frac{\partial}{\partial x_{\mathrm{j}}}\left[\eta^{\prime}\left(\frac{\partial u_{\mathrm{i}}^{*}}{\partial x_{\mathrm{j}}}+\frac{\partial u_{\mathrm{j}}^{*}}{\partial x_{\mathrm{i}}}\right)\right]+g_{\mathrm{i}} \\
\frac{\rho^{*}-\rho^{\mathrm{n}}}{\Delta t}=-\rho^{\mathrm{n}}\left(\frac{\partial u_{\mathrm{k}}^{*}}{\partial x_{\mathrm{k}}}\right) \\
\frac{p^{*}-p^{\mathrm{n}}}{\Delta t}=-\rho^{\mathrm{n}} C_{\mathrm{s}}^{2}\left(\frac{\partial u_{\mathrm{k}}^{*}}{\partial x_{\mathrm{k}}}\right)
\end{gathered}
$$

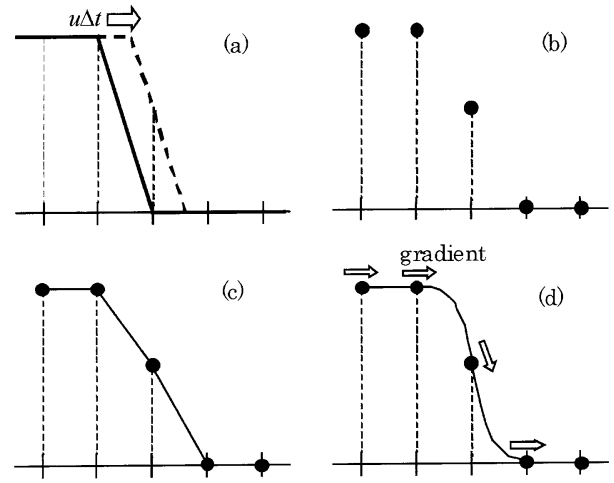

Fig. 1. Principal of CIP method

$$
\frac{\phi^{*}-\phi^{\mathrm{n}}}{\Delta t}=0
$$

In the above equations, the superscript $n$ stands for the quantities at the present time step and superscript * means one time step after the non-advection phase, and the quantities having this superscript will be used in the advection phase. In previous studies (Hadush et al., 2001; Moriguchi et al., 2002), due to the large gradients in the sound speeds of different materials, the term related to the pressure was solved implicitly and the other terms were solved by explicit procedures. In this study, however, due to the same reasons of large spatial gradients in the viscosities, an implicit procedure is used to calculate the velocity term as well. For treating the pressure term and velocity term with an implicit procedure, it is necessary to solve matrix. In this study, the Preconditioned Conjugate Gradient Squared (PCGS) method with the ILU (Incomplete LU) pre-conditioner is used to solve the finite difference matrix.

\section{Advection Phase}

$$
\begin{aligned}
& \frac{\partial u_{\mathrm{i}}}{\partial t}+u_{\mathrm{j}} \frac{\partial u_{\mathrm{i}}}{\partial x_{\mathrm{j}}}=0 \\
& \frac{\partial \rho}{\partial t}+u_{\mathrm{j}} \frac{\partial \rho}{\partial x_{\mathrm{j}}}=0 \\
& \frac{\partial p}{\partial t}+u_{\mathrm{j}} \frac{\partial p}{\partial x_{\mathrm{j}}}=-\rho C_{\mathrm{s}}^{2}\left(\frac{\partial u_{\mathrm{k}}}{\partial x_{\mathrm{k}}}\right) \\
& \frac{\partial \phi}{\partial t}+u_{\mathrm{j}} \frac{\partial \phi}{\partial x_{\mathrm{j}}}=0
\end{aligned}
$$

Equations (20), (21), (22) and (23) show advection phases of the governing equations. After calculating the non-advection phases, the CIP solver is used to solve advection phases and quantities at the next time step $\rho^{\mathrm{n}+1}$, $u_{\mathrm{i}}^{\mathrm{n}+1}, p^{\mathrm{n}+1}$ and $\phi^{\mathrm{n}+1}$ are obtained.

Figure 1 summarizes the principle of the CIP method. Let us consider a one dimensional linear advection equation:

$$
\frac{\partial f}{\partial t}+u \frac{\partial f}{\partial x}=0
$$


where $f$ stands for the quantities of density, velocity and pressure. The solution of Eq. (24) gives a simple transverse motion of wave with a velocity $u$. The initial profile (solid line of Fig. 1(a)) moves like a dashed line in a continuous representation. At this time the solution at grid points is denoted by closed circles and is the same as the exact solution. If we, however, eliminate the dashed line as in Fig. 1(b), it is difficult to imagine the original profile and is natural to retrieve the original profile like that shown by solid line in Fig. 1(c). Therefore, numerical diffusion arises when a linear interpolation is used to construct the profile even with the exact solution as shown in Fig. 1(c). Figure 1(d) shows the solution by the CIP method. In the CIP method, a spatial profile within each cell is interpolated by a cubic polynomial. Differentiating Eq. (24) with a spatial variable $x$ gives:

$$
\frac{\partial g}{\partial t}+u \frac{\partial g}{\partial x}=-\frac{\partial u}{\partial x} g
$$

where $g$ stands for the spatial derivative of $f(g=\partial f / \partial x)$. By this equation the time evolution of $f$ and $g$ can be traced on the basis of Eq. (24). If $g$ propagates as shown by the arrows in Fig. 1(d), the profile after one step is limited to a specific profile. It is not difficult to imagine that by this limitation, the solution becomes very close to the original profile. If two values of $f$ and $g$ are given at two grid points, the profile between the points can be described by cubic polynomial:

$$
F(x)=a x^{3}+b x^{2}+c x+d
$$

The profile at $n+1$ step can be obtained shifting the profile by $u \Delta t$, like:

$$
\begin{aligned}
& f^{\mathrm{n}+1}=F(x-u \Delta t) \\
& g^{\mathrm{n}+1}=d F(x-u \Delta t) / d x
\end{aligned}
$$

The above described two phases complete the numerical procedure and are repeated step by step. The CIP method has been applied to simulations of various physical problems in fluid dynamics and proved to be well performing (Yabe and Aoki, 1991; Yabe et al., 1991; Yabe and Xiao, 1993; Mutsuda, 2000).

\section{Validation of Method}

Dam-break flow is a practical problem in civil engineering and its prediction is required for the design of a dam. Experiments have been undertaken to investigate dambreak flows, where a thin plate supporting a column of water is withdrawn impulsively in a vertically upward direction. This idealized two-dimensional problem of the instantaneous removal of water barrier has long been a test case for numerical simulations, e.g., Stansby et al. (1998). In practice, the release of water will be more gradual than this idealization and depends on water-soil or concrete interaction. However, the instantaneous release may be expected to give the worst case scenario and is therefore a situation of practical, as well as fundamental interest.

Hadush et al. (2001) simulated the dam-break flow experiment using the CIP method. Figure 2 shows a two-

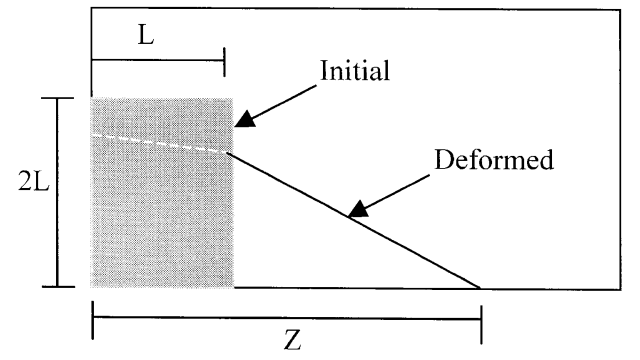

Fig. 2. Numerical model used for dam-break analysis (Hadush, 2001)

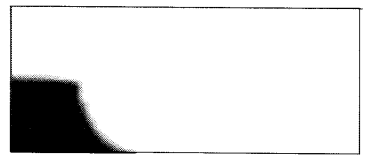

$0.1 \mathrm{~s}$

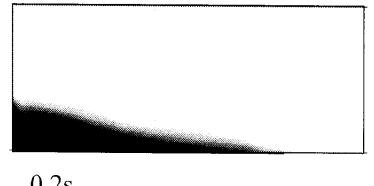

$0.2 \mathrm{~s}$
Fig. 3. Simulated surface configuration (Hadush, 2001)

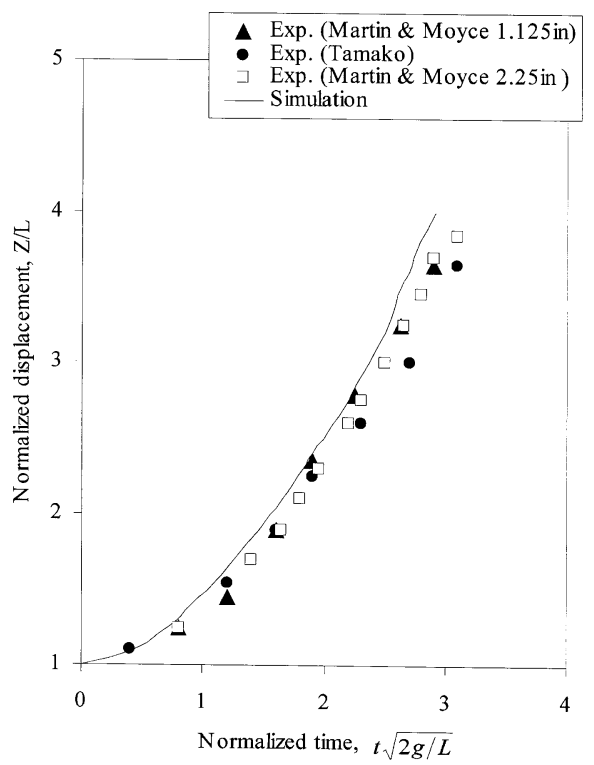

Fig. 4. Relationship between normalized time and normalized displacement (Hadush, 2001)

dimensional numerical model used in the simulation. Figure 3 shows the simulated results at two different time steps. The width of the water column, $L$, is $10 \mathrm{~cm}$ and the height is $2 L$.

Figure 4 shows the simulated time history of flow displacement, $Z$, together with the results from experiments by Martin and Moyce (1952) and Tamako (1994). As shown in this figure, both the displacement and the time are normalized by $L$. The calculated curve matches well with the results from experiments, indicating that the numerical method can be used for practical simulations.

\section{Effectiveness of Implicit Calculation}

As aforementioned, the viscous term of velocity is 


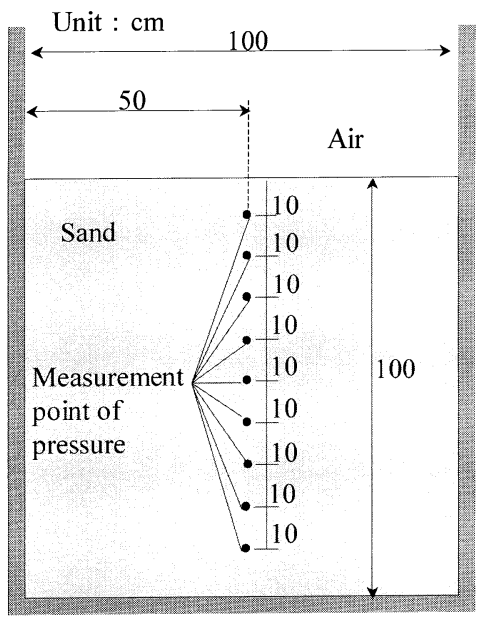

Fig. 5. Numerical model for simulation of pressure in ground

Table 1. Parameters used for simulation of pressure in ground

\begin{tabular}{ccc|c}
\hline Cohesion & $C$ & $(\mathrm{kPa})$ & 0.0 \\
\hline Internal friction angle & $\phi$ & (degree) & 30.0 \\
\hline Minimum viscosity & $\eta \quad(\mathrm{Pa} \cdot \mathrm{s})$ & 1.0 \\
\hline Time increment & $\Delta t$ & $(\mathrm{~s})$ & 0.0001 \\
\hline$X$ direction mesh size & $\Delta x \quad(\mathrm{~m})$ & 0.025 \\
\hline$Y$ direction mesh size & $\Delta y$ & $(\mathrm{~m})$ & 0.025 \\
\hline Density of geomaterial & $\rho$ & $\left(\mathrm{kg} / \mathrm{m}^{3}\right)$ & 1800.0 \\
\hline
\end{tabular}

solved implicitly. It is obvious that implicit method needs longer calculation time than explicit method but gives a more stable result. In this chapter, numerical experiments are conducted to examine the difference in numerical stabilities by explicit and implicit calculations. Figure 5 shows the two-dimensional numerical model used for the numerical experiments. Table 1 summarizes the parameters used in the simulation. The ground is assumed to be composed of a granular material and gravity is the only external force in this simulation. The gravity is vertical to the bottom of the analytical area. The boundary conditions for side and bottom wall are slip boundary conditions. This indicates that friction is not considered at the side and bottom walls in order to minimize its effects on the results. In the initial, the ground is at stationary condition, and there is no spatial distribution of pressure. After stating calculation, the ground is subjected to gravity effects and spatial distribution of pressure arises. In the analysis, pressures at different depth are picked up as shown in Fig. 5. The theoretical pressure distribution in the ground is described as below,

$$
p=\rho g h
$$

where $g$ is the gravity acceleration and $h$ is the depth below surface of the ground.

Figures 6 and 7 show the simulated results for explicit

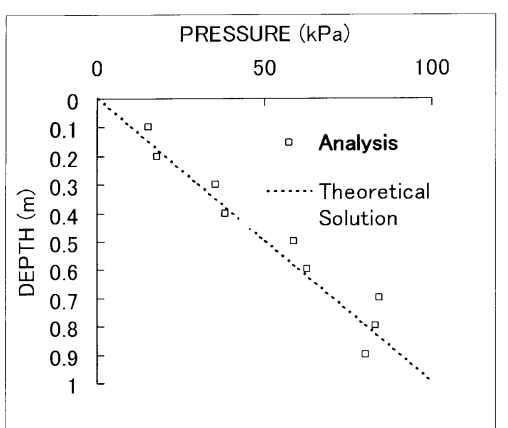

Fig. 6. Relationship between depth and pressure by explicit calculation

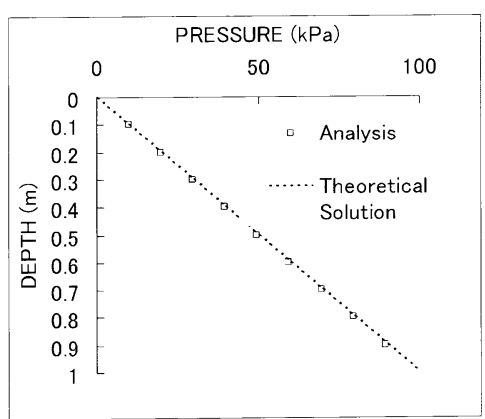

Fig. 7. Relationship between depth and pressure by implicit calculation

Table 2. Parameters used for simulation of gravitational flow

\begin{tabular}{c|c|c|c|c}
\hline & Case 1 & Case 2 & Case 3 & Case 4 \\
\hline$C(\mathrm{kPa})$ & 0.0 & 0.0 & 4.0 & 6.0 \\
\hline$\phi($ degree $)$ & 30.0 & 60.0 & 0.0 & 0.0 \\
\hline$\eta(\mathrm{Pa} \cdot \mathrm{s})$ & & 1.0 & \\
\hline$\Delta x(\mathrm{~m})$ & & 0.1 \\
\hline$\Delta y(\mathrm{~m})$ & 0.1 \\
\hline$\rho\left(\mathrm{kg} / \mathrm{m}^{3}\right)$ & 1800.0 \\
\hline
\end{tabular}

and implicit methods together with theoretical solutions, respectively. From these figures, it is found that the result from the implicit calculation is in a very good agreement with the theoretical solutions, and that implicit procedure is necessary to treat Bingham viscosity in the simulation. By the way, the result from explicit calculation (Fig. 6) shows very interesting pressure distribution. The reason for this interesting pressure distribution is considered as follows. In this simulation, as mentioned above, the ground is at stationary condition in the initial, and the gravity affects after stating calculation. Therefore, the calculation is in an unstable condition, and Bingham viscosity changes greatly with space and time. The explicit calculation can not treat this rapid change of Bingham viscosity. Therefore, interesting pressure distribution shown in Fig. 6 was obtained. 
Table 3. Parameters used for simulation of slump test

\begin{tabular}{cl|c|c|c}
\hline \multicolumn{1}{|c|}{} & & Case 1 & Case 2 & Case 3 \\
\hline$\phi$ & $(\mathrm{kPa})$ & 0.0 & 1.0 & 2.0 \\
\hline$\eta$ & $(\mathrm{degree})$ & 5.7 & 5.7 & 5.7 \\
\hline$\Delta x$ & $(\mathrm{~m})$ & 1.0 & \\
\hline$\Delta y$ & $(\mathrm{~m})$ & 0.01 \\
\hline$\rho$ & $\left(\mathrm{kg} / \mathrm{m}^{3}\right)$ & & 0.01 \\
\hline
\end{tabular}

Table 4. Parameters used for simulation of landslide

\begin{tabular}{cl|c}
\hline$C$ & $(\mathrm{kPa})$ & 1.0 \\
\hline$\phi$ & $($ degree $)$ & 5.7 \\
\hline$\eta \quad(\mathrm{Pa} \cdot \mathrm{s})$ & 1.0 \\
\hline$\Delta x \quad(\mathrm{~m})$ & 2.104 \\
\hline$\Delta y \quad(\mathrm{~m})$ & 0.526 \\
\hline$\rho$ & $\left(\mathrm{kg} / \mathrm{m}^{3}\right)$ & 1800.0 \\
\hline
\end{tabular}

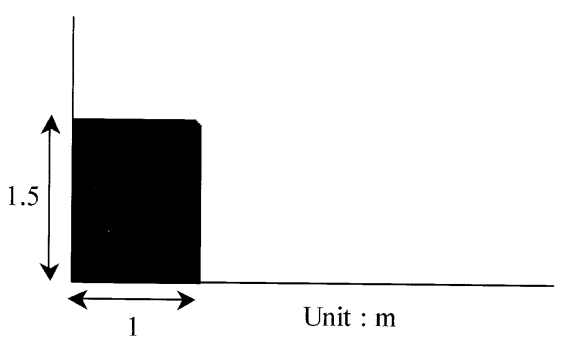

Fig. 8. Initial condition for simulation of gravitational flow

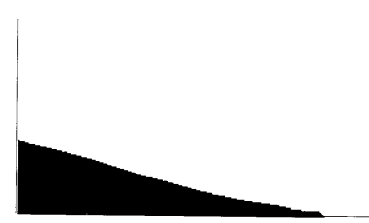

a) Case1 $\left(\phi=30^{\circ}\right)$

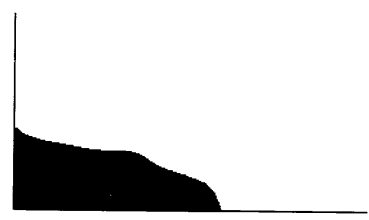

c) Case $3 \quad(\mathrm{c}=4 \mathrm{kPa})$

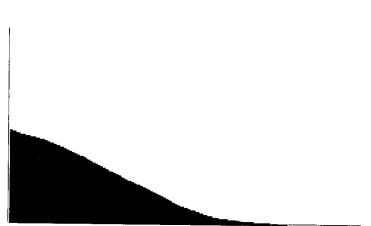

b) Case2 $\left(\phi=60^{\circ}\right)$

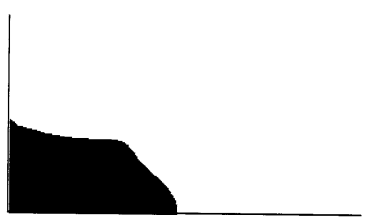

d) Case4 $\quad(\mathrm{c}=6 \mathrm{kPa})$
Fig. 9. Simulated final surface configurations for different $c$ and $\phi$

\section{Model Performance (Gravitational Flow of a Geomateri- al Column)}

Bingham model is used as a basic constitutive model taking into consideration the shear strength of soils governed by cohesion $c$ and friction angle $\phi$. In this chapter, gravitational flow of a geomaterial is simulated

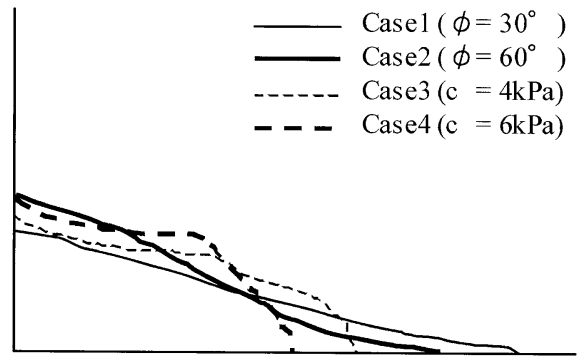

Fig. 10. Final surface configurations of all cases together

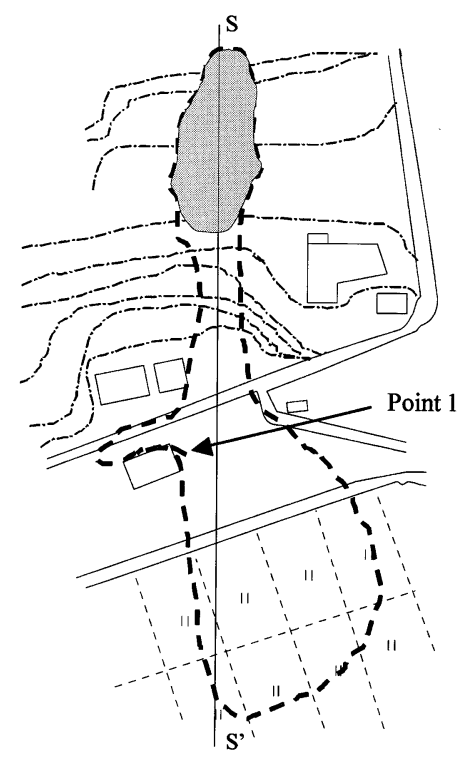

Fig. 11. Schematic diagram of flowed area

to investigate the deformation behavior and performance of the constitutive model. Four cases with different parameters are considered for this simulation. Table 2 summarizes the parameters used in the simulations. In cases 1 and 2, the ground is assumed to be composed of granular cohesionless geomaterials, and in cases 3 and 4 composed of cohesive geomaterials. Figure 8 shows the initial condition for these simulations. The soil column is $1.5 \mathrm{~m}$ in height and $1 \mathrm{~m}$ in length. The left and bottom walls have non-slip boundary conditions. Simulated final surface consignations are shown in Fig. 9 where a, b, c and $\mathrm{d}$ are the results from cases $1,2,3$, and 4 , respectively. Figure 10 shows simulated final surface configurations for all cases together. It is found that in the case of granular geomaterials, after flow, the ground stops with a certain reposing angle, which is coincident to the internal friction angle $\phi$. In the case of the cohesive geomaterials, the ground stands almost vertically which is a typical behavior of cohesive soils.

\section{SIMULATION OF LANDSLIDE}

The numerical method developed in this study is finally used to simulate a high-speed landslide that took place on May 26th, 2003 in the Northern part of Miyagi Prefecture 
in Japan. The detailed description of the incident was introduced in the report of Sanriku-Minami earthquake (The Japanese Geotechnical Society, 2003). The landslide took place at about a $27 \mathrm{~m}$ high hill composed of volcanic deposits. According to local residents, the ground movement lasted from $100 \mathrm{~s}$ to $120 \mathrm{~s}$. Konagai (2003) reported that the sliding slope was $40 \mathrm{~m}$ wide, $60 \mathrm{~m}$ long. The sliding distance was $180 \mathrm{~m}$. The maximum velocity at a boundary point (Point 1 in Fig. 11) of the sliding slope was reported to be 3.3 to $3.6 \mathrm{~m} / \mathrm{s}$; and from relative movement of trees, the velocity at the center was estimated to be 6 to $7 \mathrm{~m} / \mathrm{s}$. The ground consisted of $20 \%$ boulder, $50 \%$ sand, $20 \%$ silt, $10 \%$ clay and the water

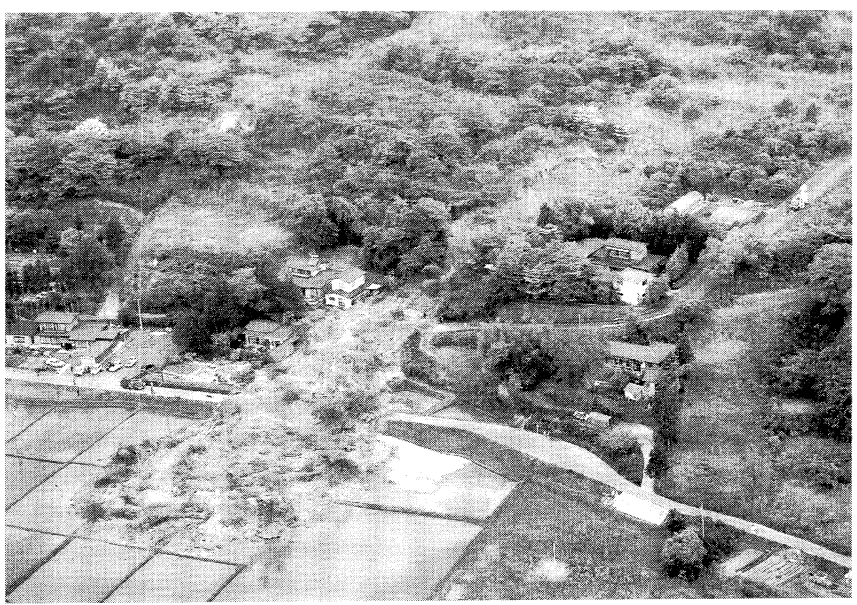

Photo 1. Aerial photograph of the landslide

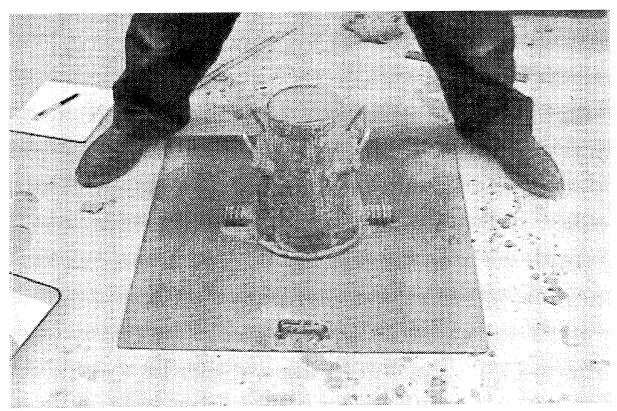

Photo 2. Sample of soil-slump test

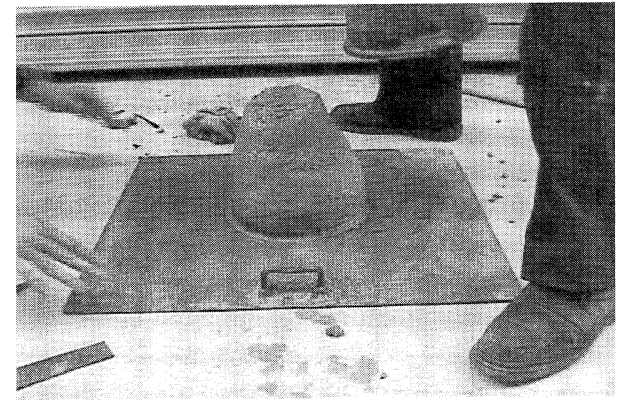

Case 1 content was as high as $26 \%$ to $56 \%$. Photo 1 shows an aerial photograph of the landslide site and Fig. 11 shows a schematic view of the sliding slope together with the ground flow and displacement region.

\section{Soil-Slump Tests}

In this study, soil-slump tests are conducted on samples obtained from the landslide site soon after the incident. This experiment is based on the method used for concrete testing (JIS A 1101) and as shown in Photo 2 and Fig. 12. The samples were $30 \mathrm{~cm}$ in height and between $10 \mathrm{~cm}$ (top) to $20 \mathrm{~cm}$ (bottom) in diameter. Two cases: 1) sample with $42.5 \%$ water content and 2) sample with $47.5 \%$ water content were used in the experiments. As shown in Photo 3, the slump value was $8.5 \mathrm{~cm}$ for case 1 and $23.5 \mathrm{~cm}$ for case 2 , respectively. After the slump, the final bottom diameter was $20 \mathrm{~cm}$ for case 1 and $47 \mathrm{~cm}$ for case

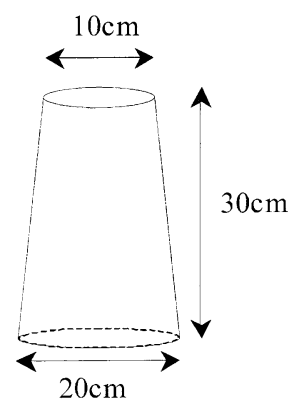

Fig. 12. Size of sample used for soil-slump test

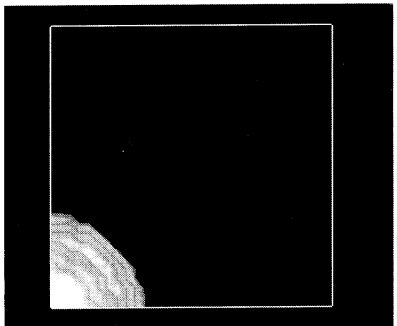

(a) View from side

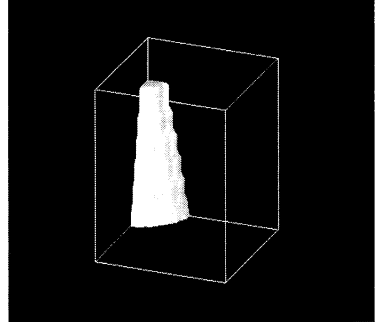

(b) View from top
Fig. 13. Initial surface configuration of numerical model for simulation of soil slump test

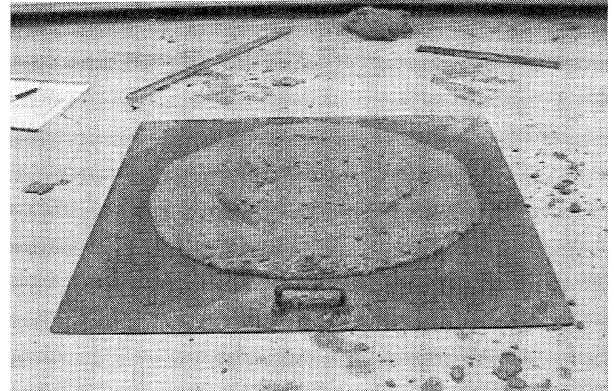

Case2

Photo 3. Final surface configuration (Case 1, 2) 


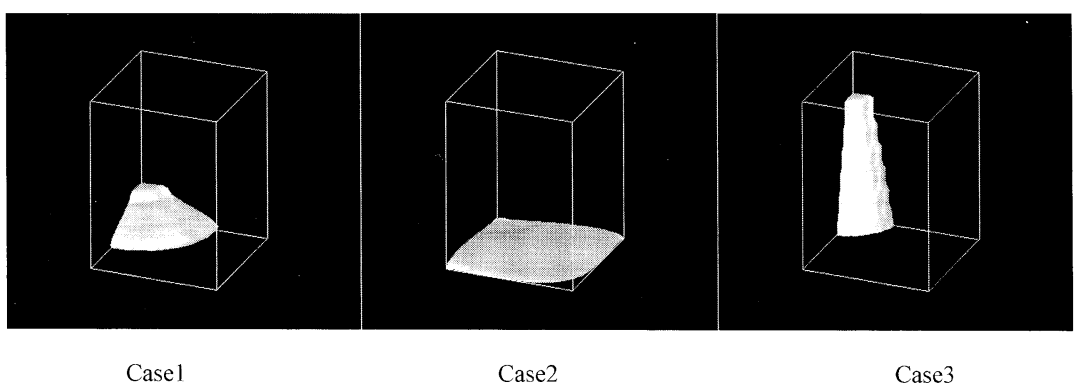

Fig. 14. Simulated final surface configurations of sample (Case 1, 2, 3)

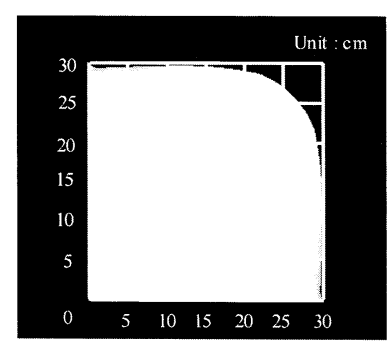

Case 1

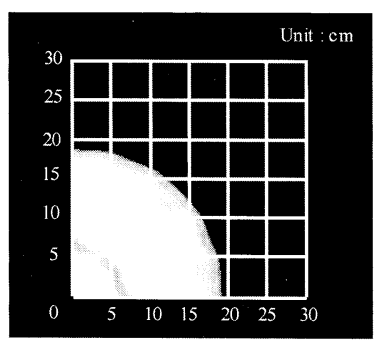

Case2

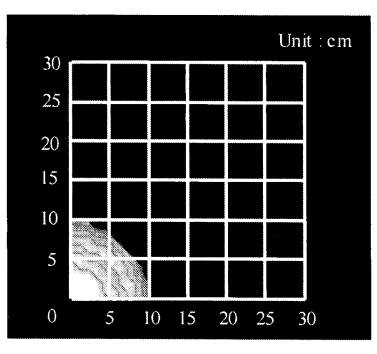

Case3

Fig. 15. Simulated final surface configurations viewed from top (Case 1, 2, 3)

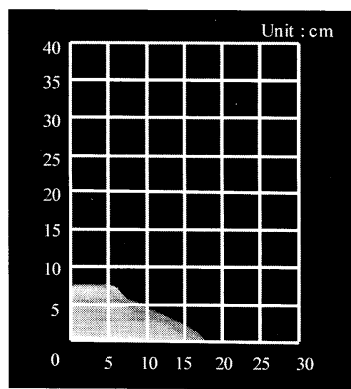

Fig. 16. Final surface configuration viewed from side (Case 2)

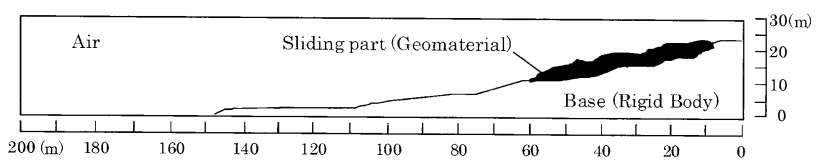

Fig. 17. Numerical model for simulation of landslide

2. These results clearly indicate that the water content largely affects the behavior of the soil.

\section{Simulation of Soil-Slump Tests}

To determine the parameters for the landslide analysis, a 3-dimensional simulation of the soil slump tests were carried out. Figure 13 shows the numerical model used in this simulation viewed from side and top. Due to symmetric configuration, only one fourth of the sample shape is used in the simulation in order to reduce calculation time. According to the results of the soil-slump tests, the behavior of the sample greatly depends on water content. In order to reflect the influence of water content in the behavior of soil slump, three cases with different cohesion

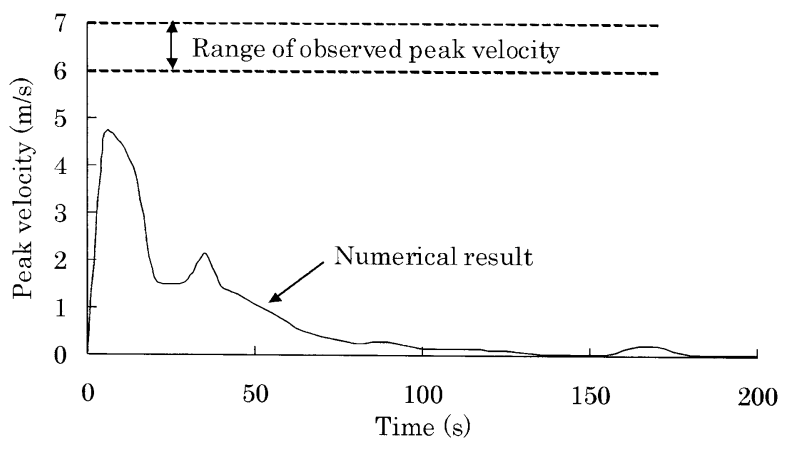

Fig. 18. Simulated time history of peak velocity

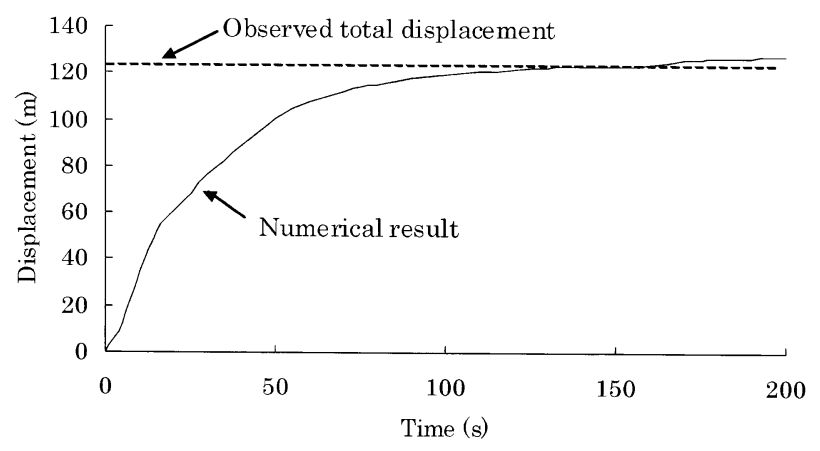

Fig. 19. Simulated time history of displacement

values were considered in the calculations. It is known that cohesion, which by strictly speaking, is merely an apparent one, is affected by the water content. As to the internal friction angle, constant value is used, and the internal friction angle is estimated from slope angle of the landslide site. Figures 14 and 15 show the simulated 

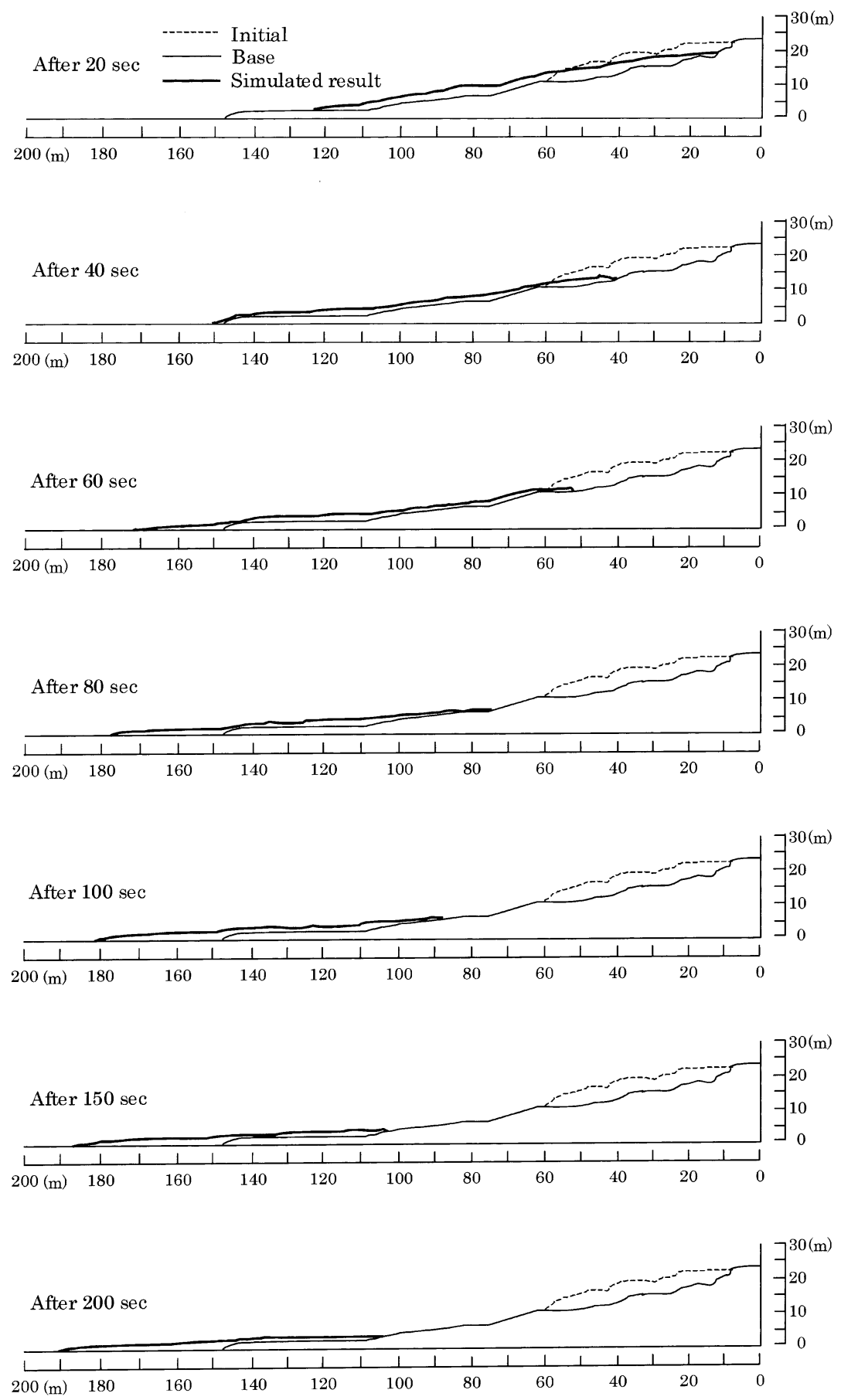

Fig. 20. Simulated surface configurations

results viewed from side and top, respectively. In case 1, the slump does not stop until it reaches a rigid boundary. In case 2 , the slump stops as shown in Fig. 16 with a slump value of $22 \mathrm{~cm}$ and the final bottom diameter in this case was $18 \mathrm{~cm}$. In case 3 , the soil does not slump at all. Therefore, because the results from case 2 simulation are in good agreement with the test results, the parameters used in case 2 are selected for the landslide simulation.

\section{Simulation of Actual Landslide}

It is assumed that there is a difference between results from two-dimensional analysis and three-dimensional analysis. As seen in Photo 1, however, there is not large inequality of the ground in the landslide site, and the ground can be seen like planar slope with small slope angle. Therefore, it can be considered that two-dimensional analysis can predict with a certain level of accuracy. For this reason, the simulation is carried out in twodimensional. From topographic surveying data and related maps, we prepared a model to simulate the landslide. Figure 17 shows a two-dimensional numerical model used for the simulation of the landslide.

Figures 17 and 18 show the simulated time history of 


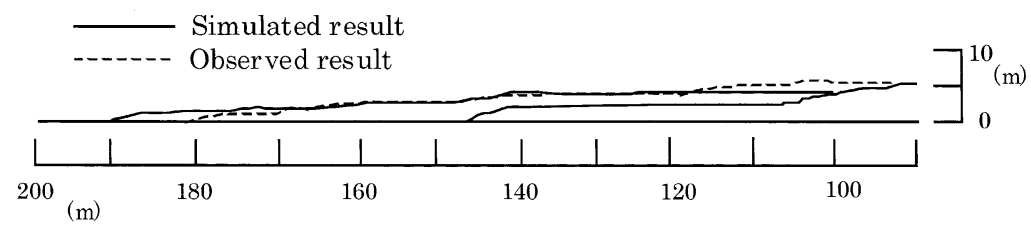

Fig. 21. Final surface configuration

peak velocity and displacement, respectively. In order to compare the numerical results with observed results, observed peak velocity and total displacement of the real ground flow are described in Figs. 17 and 18. In this simulation the peak velocity corresponds to the maximum velocity that occurred in the analytical domain at each calculation step. The total displacement is defined as the distance from the initial front of the sliding part. In this case, the observed total displacement is equal to $123 \mathrm{~m}$. Figure 20 shows simulated surface configurations at different times. Figure 21 shows the final ground profiles. As shown in these figures, simulated results are in good agreement with the observed results. There is, however, still minor discrepancy between the simulated and the observed results. Following are considered as reasons for this minor discrepancy. First reason is that two-dimensional analysis cannot perfectly predict a real three-dimensional phenomenon. The second reason being, change in the volume of geomaterials due to dilatancy and material compressive is not considered in the proposed method. Third reason is that geomaterials are treated as a single-fluid in the method. For this reason, average parameters are used in simulations, and heterogeneity of geomaterials is not mentioned. Final reason is the parameter uncertainty; that the parameters used in this simulation were determined from only results of soil-slump tests.

Although the proposed method still has some problem as mentioned above, it is found that the method is effective to simulate flow failure of geomaterials.

\section{CONCLUSIONS}

This work presented a fluid dynamics based numerical method for predicting large deformations of geomaterials during landslide or slope failure. A Bingham-type constitutive model formulated based on Coulomb's failure criterion was used to represent the viscous behavior of the geomaterial and the viscosity was estimated from cohesion and friction angle. The results from this work are written up as follows.

1) Numerical experiments are conducted to examine the difference in numerical stabilities by explicit and implicit calculations. It is found that an implicit calculation is very effective for the simulation using Bingham model.

2) Numerical experiments on a $2 \mathrm{D}$ gravitational flow of a geomaterials column showed that the constitutive model performs well. It is found that the Bingham model can describe typical behaviors of both granular and cohesive soils.
3) To determine the parameters for landslide analysis, a 3D simulation of soil-slump test was carried out. Not only the parameters used for numerical analysis can be determined easily, but also the slope stability can be well predicted by the method.

4) The method was also used to simulate an earthquakeinduced landslide. Although the proposed method still has some problem as mentioned above, it is found that the method is effective to simulate flow failure of geomaterials.

\section{REFERENCES}

1) Belytschko, T., Lu, Y. Y. and Gu, L. (1994): Element free galerkin methods, Int. J. Numer. Methods Eng., 37, 229-256.

2) Hadush, S., Yashima, A. and Uzuoka, R., (2000): Importance of viscous fluid characteristics in liquefaction induced lateral spreading analysis, Computers and Geotechnics, 27, 199-224.

3) Hadush, S., Yashima, A., Uzuoka, R., Moriguchi, S. and Sawada, K. (2001): Liquefaction induced lateral spread analysis using the CIP method, Computers and Geotechnics, 28, 549-574.

4) Hamada, M. and Wakamatsu, K. (1998): A study on ground displacement caused by soil liquefaction, J. Geotechnical Engineering, JSCE, 596, 189-208 (in Japanese).

5) Huerta, A. and Liu, W. K. (1988): Viscous flow with large free surface motion, Comput. Meth. Appl. Mesh. Eng., 69, 277-324.

6) Konagai, K. (2003): Some challenges in earthquake damage surveys, Tsuchi-to-Kiso, The Japanese Geotechnical Society, 52, 16-18 (in Japanese).

7) Martin, J. C. and Moyce, W. J. (1952): An experimental study of the collapse of liquid column on a rigid horizontal plane, Trans. $R$. Soc. London Ser. A, 244, 312-324.

8) Moriguchi, S., Yashima, A. and Sawada, K. (2002): Large deformation analysis of geomaterials in the frame work of fluid dynamics using CIP method, Proc. 1st Int. Workshop on New Frontiers in Computational Geotechnics, Banff Alberta, Canada, 107-110.

9) Mutsuda, H. (2000): A highly accurate numerical scheme for a fully developed turbulent flow caused by large scale breakers, Ph.D. Dissertation, Gifu University, Japan (in Japanese).

10) Sakaguchi, T. and Yoshida, M. (1998): An experimental and analytical study on characteristics of flow and external force of liquefied ground, Masters Dissertation, Waseda University, Japan (in Japanese).

11) Stansby, P. K., Chegini, A. and Barnes, T. C. D. (1998): The initial stages of dam-break flow, J. Fluid Mech., 370, 203-220.

12) Tamako, H. (1994): Development of particle method for the dynamics of free boundaries, Master Thesis, Tokyo University, Tokyo (in Japanese).

13) The Japanese Geotechnical Society (2003): Report of Seismic Hazard of Sanriku-Minami Earthquake and Miyagi Prefecture North Earthquake (in Japanese).

14) Uzuoka, R. (2000): Analytical study on the mechanical behavior and prediction of soil liquefaction and flow, Ph.D. Dissertation, Gifu University, Japan (in Japanese).

15) Uzuoka, R., Yashima, A., Kawakami, T. and Konrad, J.-M. (1998): Fluid dynamics based prediction of liquefaction induced 
lateral spreading, Computers and Geotechnics, 22(3/4), 234-282.

16) Xiao, F. (1999): A computational model for suspended large rigid body in 3D unsteady viscous flow, J. Comput. Phys., 155.

17) Yabe, T. and Aoki, T. (1991): A universal solver for hyperbolic equations by cubic polynomial interpolation, Comput. Phys. Commun., 66, 219-232.

18) Yabe, T. and Xiao, F. (1993): Description of complex and sharp interface during shock wave interaction with liquid drop, J. Phys. Soc., Japan, 62, 2537-2540.

19) Yabe, T., Ishikawa, T., Wang, P. Y., Aoki, T., Kodata, Y. and Ikeda, F. (1991): A universal solver for hyperbolic equations by cubic-polynomial interpolation, two- and three-dimensional solvers, Comput. Phys. Commun., 66, 233-242. 